\title{
A Note on the Sequence Related to Catalan Numbers
}

\author{
Jin Zhang ${ }^{1}$ and Zhuoyu Chen ${ }^{2, *}$
}

1 School of Information Engineering, Xi'an University, Xi'an 710127, China; zhangjin0921@xawl.edu.cn

2 School of Mathematics, Northwest University, Xi'an 710127, China

* Correspondence: chenzymath@stumail.nwu.edu.cn

Received: 7 February 2019; Accepted: 9 March 2019; Published: 13 March 2019

Abstract: The main purpose of this paper is to find explicit expressions for two sequences and to solve two related conjectures arising from the recent study of sums of finite products of Catalan numbers by Zhang and Chen.

Keywords: new sequence; Catalan numbers; elementary and combinatorial methods; congruence; conjecture

MSC: 11B83; 11B75

\section{Introduction}

Let $n$ be any non-negative integer. Then, $C_{n}=\frac{1}{n+1} \cdot\left(\begin{array}{c}2 n \\ n\end{array}\right)(n=0,1,2,3, \cdots)$ are defined as the Catalan numbers. For example, the first several values of the Catalan numbers are $C_{0}=1, C_{1}=1$, $C_{2}=2, C_{3}=5, C_{4}=14, C_{5}=42, C_{6}=132, C_{7}=429, C_{8}=1430, \cdots$. The generating function of the sequence $\left\{C_{n}\right\}$ is:

$$
\frac{2}{1+\sqrt{1-4 x}}=\sum_{n=0}^{\infty} \frac{\left(\begin{array}{c}
2 n \\
n
\end{array}\right)}{n+1} \cdot x^{n}=\sum_{n=0}^{\infty} C_{n} \cdot x^{n}
$$

This sequence occupies a pivotal position in combinatorial mathematics, so lots of counting problems are closely related to it. A great number of examples can be found in a study by Stanley [1]. Because of these, plenty of scholars have researched the properties of Catalan numbers and obtained a large number of vital and meaningful results. Interested readers can refer to the relevant references [2-26], which is not an exhaustive list. Very recently, Zhang and Chen [27] researched the calculation problem of the following convolution sums:

$$
\sum_{a_{1}+a_{2}+\cdots+a_{h}=n} C_{a_{1}} \cdot C_{a_{2}} \cdot C_{a_{3}} \cdots C_{a_{h}}
$$

where the summation has taken over all $h$-dimension non-negative integer coordinates $\left(a_{1}, a_{2}, \cdots, a_{h}\right)$, such that the equation $a_{1}+a_{2}+\cdots+a_{h}=n$.

They first introduced two new recursive sequences, $C(h, i)$ and $D(h, i)$, and after the elementary and combinatorial methods, they proved the following two significant conclusions:

Theorem 1. For any positive integer $h$, one gets the identity:

$$
\begin{gathered}
\sum_{a_{1}+a_{2}+\cdots+a_{2 h+1}=n} C_{a_{1}} \cdot C_{a_{2}} \cdot C_{a_{3}} \cdots C_{a_{2 h+1}} \\
=\frac{1}{(2 h) !} \sum_{i=0}^{h} C(h, i) \sum_{j=0}^{\min (n, i)} \frac{(n-j+h+i) ! \cdot C_{n-j+h+i}}{(n-j) !} \cdot\left(\begin{array}{l}
i \\
j
\end{array}\right) \cdot(-4)^{j},
\end{gathered}
$$


where the sequence $C(h, i)$ is defined as $C(1,0)=-2, C(h, h)=1, C(h+1, h)=C(h, h-1)-(8 h+$ $2) \cdot C(h, h), C(h+1,0)=8 \cdot C(h, 1)-2 \cdot C(h, 0)$, and for all integers $1 \leq i \leq h-1$, we acquire the recursive formula:

$$
C(h+1, i)=C(h, i-1)-(8 i+2) \cdot C(h, i)+(4 i+4)(4 i+2) \cdot C(h, i+1) .
$$

Theorem 2. For any positive integer $h$ and non-negative $n$, one can obtain:

$$
\begin{gathered}
\sum_{a_{1}+a_{2}+\cdots+a_{2 h}=n} C_{a_{1}} \cdot C_{a_{2}} \cdot C_{a_{3}} \cdots C_{a_{2 h}} \\
=\frac{1}{(2 h-1) !} \sum_{i=0}^{h-1} \sum_{j=0}^{n} D(h, i+1) \cdot\left(\begin{array}{c}
i+\frac{1}{2} \\
j
\end{array}\right) \cdot(-4)^{j} \cdot \frac{(n-j+h+i) ! \cdot C_{n-j+h+i}}{(n-j) !},
\end{gathered}
$$

where $\left(\begin{array}{c}n+\frac{1}{2} \\ i\end{array}\right)=\left(n+\frac{1}{2}\right) \cdot\left(n-1+\frac{1}{2}\right) \cdots\left(n-i+1+\frac{1}{2}\right) / i$ !, the sequence $D(k, i)$ are defined as $D(k, 0)=0, D(k, k)=1, D(k+1, k)=D(k, k-1)-(8 k-2), D(k+1,1)=24 D(k, 2)-6 D(k, 1)$, and for all integers $1 \leq i \leq k-1$,

$$
D(k+1, i)=D(k, i-1)-(8 i-2) \cdot D(k, i)+4 i(4 i+2) \cdot D(k, i+1) .
$$

Meanwhile, through numerical observation, Zhang and Chen [27] also proposed the following two conjectures:

Conjecture 1. Let $p$ be a prime. Then, for any integer $0 \leq i<\frac{p+1}{2}$, we obtain the congruence:

$$
C\left(\frac{p+1}{2}, i\right) \equiv 0 \bmod p(p+1) \text {. }
$$

Conjecture 2. Let $p$ be a prime. Then, for any integer $0 \leq i<\frac{p+1}{2}$, we obtain the congruence:

$$
D\left(\frac{p+1}{2}, i\right) \equiv 0 \bmod p(p-1) \text {. }
$$

For easy comparison, here we list some of the values of $C(h, i)$ and $D(h, i)$ with $1 \leq h \leq 6$ and $0 \leq i \leq h$ in the following Tables 1 and 2 .

Table 1. Values of $C(k, i)$.

\begin{tabular}{cccccccc}
\hline$C(\boldsymbol{k}, \boldsymbol{i})$ & $\boldsymbol{i = 0}$ & $\boldsymbol{i = 1}$ & $\boldsymbol{i = 2}$ & $\boldsymbol{i = 3}$ & $\boldsymbol{i = 4}$ & $\boldsymbol{i = 5}$ & $\boldsymbol{i = 6}$ \\
\hline $\boldsymbol{k}=\mathbf{1}$ & -2 & 1 & & & & & \\
$\boldsymbol{k}=\mathbf{2}$ & 12 & -12 & 1 & & & & \\
$\boldsymbol{k}=\mathbf{3}$ & -120 & 180 & -30 & 1 & & & \\
$\boldsymbol{k}=\mathbf{4}$ & 1680 & -3360 & 840 & -56 & 1 & & \\
$\boldsymbol{k}=\mathbf{5}$ & $-30,240$ & 75,600 & $-25,200$ & 2520 & -90 & 1 & \\
$\boldsymbol{k}=\mathbf{6}$ & 665,280 & $-1,995,840$ & 831,600 & $-110,880$ & 5940 & -132 & 1 \\
\hline
\end{tabular}

Table 2. Values of $D(k, i)$.

\begin{tabular}{cccccccc}
\hline $\boldsymbol{D}(\boldsymbol{k}, \boldsymbol{i})$ & $\boldsymbol{i}=\mathbf{0}$ & $\boldsymbol{i = 1}$ & $\boldsymbol{i = 2}$ & $\boldsymbol{i = 3}$ & $\boldsymbol{i = 4}$ & $\boldsymbol{i = 5}$ & $\boldsymbol{i}=\mathbf{6}$ \\
\hline $\boldsymbol{k}=\mathbf{1}$ & 0 & 1 & & & & & \\
$\boldsymbol{k}=\mathbf{2}$ & 0 & -6 & 1 & & & & \\
$\boldsymbol{k}=\mathbf{3}$ & 0 & 60 & -20 & 1 & & & \\
$\boldsymbol{k}=\mathbf{4}$ & 0 & -840 & 420 & -42 & 1 & & \\
$\boldsymbol{k}=\mathbf{5}$ & 0 & 15,120 & $-10,080$ & 1512 & -72 & 1 & \\
$\boldsymbol{k}=\mathbf{6}$ & 0 & -332640 & 277,200 & $-55,440$ & 3960 & -110 & 1 \\
\hline
\end{tabular}


Based on these two tables and a large number of numerical calculations, we found that these conjectures are not only correct, but also have generalized conclusions. Actually, they provide a simpler and clearer representation.

In this paper, by using some notes from Zhang and Chen's work [27] as well as some basic and combinatorial methods, we are going to prove the following:

Theorem 3. Let $h$ be a positive integer. Then, for any integer $i$ with $0 \leq i \leq h$, we acquire the identity:

$$
C(h, i)=(-1)^{h-i} \cdot \frac{(2 h) !}{(h-i) ! \cdot(2 i) !} .
$$

Theorem 4. Let $h$ be a positive integer. Then, for any integer $i$ with $1 \leq i \leq h$, we acquire the identity:

$$
D(h, i)=(-1)^{h-i} \cdot \frac{(2 h-1) !}{(h-i) ! \cdot(2 i-1) !} .
$$

Based on the above two theorems, we may instantly deduce the following two corollaries:

Corollary 1. Let $h$ be any positive integer. Then, for any integer $0 \leq i \leq h-1$, we gain the congruence:

$$
C(h, i) \equiv 0 \bmod 2 h(2 h-1) .
$$

Corollary 2. Let $h$ be any positive integer. Then, for any integer $0 \leq i \leq h-1$, we gain the congruence:

$$
D(h, i) \equiv 0 \bmod (2 h-1)(2 h-2) .
$$

Suppose that we consider $p$ an odd prime, and that when $h=\frac{p+1}{2}$ in Corollary 1 and Corollary 2, combined with the identities $2 h(2 h-1)=p(p+1)$ and $(2 h-1)(2 h-2)=p(p-1)$, our Corollary 1 and Corollary 2 proves Conjecture 1 and Conjecture 2, respectively. Practically, they prove two more general conclusions.

Taking $n=0$ in Theorem 1 and Theorem 2 and applying our theorems, we may instantly deduce the following two identities:

Corollary 3. Let $h$ be any positive integer. Then, we get the identity:

$$
\sum_{i=0}^{h}(-1)^{h-i}\left(\begin{array}{c}
h+i \\
2 i
\end{array}\right) \cdot C_{h+i}=1 .
$$

Corollary 4. Let $h$ be any positive integer. Then, we get the identity:

$$
\sum_{i=1}^{h}(-1)^{h-i}\left(\begin{array}{c}
h+i-1 \\
2 i-1
\end{array}\right) \cdot C_{h+i-1}=1
$$

Some notes: If we replace $C(h, i)(D(h, i))$ in Theorem 1 (Theorem 2) with the formula for $C(h, i)$ $(D(h, i))$ in our Theorem 3 (Theorem 4$)$, then we can get a more accurate representation for convolution sums (2).

The proof of the results in this paper is uncomplicated, but guessing their specific forms is not easy.

\section{Proofs of the Theorems}

Actually, the recursive form of the sequence $C(h, i)$ or $D(h, i)$ is more complex, but as long as we are able to guess its accurate representation, it is not difficult to prove. First of all, combining the mathematical induction method, we are going to prove: 


$$
C(h, i)=(-1)^{h-i} \cdot \frac{(2 h) !}{(h-i) ! \cdot(2 i) !} .
$$

According to Table 1, we know that $C(1,0)=-2, C(1,1)=1, C(2,0)=-12, C(2,1)=12$, $C(2,2)=1, C(3,0)=-120, C(3,1)=180, C(3,2)=-30, C(3,3)=1$. This means that $(3)$ is correct for $h=1,2,3$, and $0 \leq i \leq h$.

Assume that (3) is correct for integer $h=k$ and all $0 \leq i \leq k$. That is,

$$
C(k, i)=(-1)^{k-i} \cdot \frac{(2 k) !}{(k-i) ! \cdot(2 i) !}, 0 \leq i \leq k .
$$

Then, for $h=k+1$, if $i=h+1$, applying the definition of $C(h, i)$, we acquire $C(k+1, k+1)=1$. If $i=0$, combining the inductive hypothesis (4) and noting that $C(k+1,0)=8 C(k, 1)-2 C(k, 0)$, we obtain:

$$
C(k+1,0)=8 \cdot(-1)^{k-1} \cdot \frac{(2 k) !}{(k-1) ! \cdot 2 !}-(-1)^{k} \cdot 2 \cdot \frac{(2 k) !}{k !}=(-1)^{k+1} \frac{(2 k+2) !}{(k+1) !} .
$$

Suppose that $1 \leq i \leq k$. From (4) and the recursive properties of $C(h, i)$, we gain:

$$
\begin{aligned}
& C(k+1, i)=C(k, i-1)-(8 i+2) \cdot C(k, i)+(4 i+4)(4 i+2) \cdot C(k, i+1) \\
& =(-1)^{k-i+1} \frac{(2 k) !}{(k-i+1) !(2 i-2) !}-(-1)^{k-i}(8 i+2) \frac{(2 k) !}{(k-i) !(2 i) !} \\
& +(-1)^{k-i-1}(4 i+4)(4 i+2) \cdot \frac{(2 k) !}{(k-i-1) !(2 i+2) !} \\
& =(-1)^{k+1-i} \cdot \frac{(2 k+2) !}{(k+1-i !) \cdot(2 i) !} .
\end{aligned}
$$

According to (5) and (6), we know that the Formula (3) is correct for $h=k+1$ and all integers $0 \leq i \leq k+1$. Theorem 3 can then be proved by mathematical induction.

In a similar way, we can also prove Theorem 4 by mathematical induction. Since the proof process is the same as the proof of Theorem 3, it is omitted.

\section{Conclusions}

The main purpose of this paper was to give two specific expressions for the sequences $C(h, i)$ and $D(h, i)$. As for some applications of our results, we proved two conjectures proposed by Zhang and Chen in [27].

As a matter of fact, our results are more general and not subject to prime conditions. Meanwhile, using our formulae for $C(h, i)$ and $D(h, i)$ in the theorems, we can simplify the variety of results that appear in Reference [27].

This paper not only enriches the research content of the Catalan numbers, but can also be regarded as a supplement and further improvement to Zhang and Chen's work in [27].

Author Contributions: All authors have equally contributed to this work. All authors read and approved the final manuscript.

Funding: This work is supported by the N. S. F. (11771351) and (11826205) of China.

Acknowledgments: The author would like to thank the referees for their very helpful and detailed comments, which have significantly improved the presentation of this paper.

Conflicts of Interest: The authors declare that there are no conflicts of interest regarding the publication of this paper.

\section{References}

1. Stanley, R.P. Enumerative Combinatorics: Volume 2 (Cambridge Studieds in Advanced Mathematics); Cambridge University Press: Cambridge, UK, 1997; p. 49.

2. Chu, W.C. Further identities on Catalan numbers. Discr. Math. 2018, 341, 3159-3164. [CrossRef] 
3. Anthony, J.; Polyxeni, L. A new interpretation of the Catalan numbers arising in the theory of crystals. J. Algebra 2018, 504, 85-128.

4. Liu, J.C. Congruences on sums of super Catalan numbers. Results Math. 2018, 73, 73-140. [CrossRef]

5. Kim, D.S.; Kim, T. A new approach to Catalan numbers using differential equations. Russ. J. Math. Phys. 2017, 24, 465-475. [CrossRef]

6. Kim, T.; Kim, D.S. Some identities of Catalan-Daehee polynomials arising from umbral calculus. Appl. Comput. Math. 2017, 16, 177-189.

7. Kim, T.; Kim, D.S. Differential equations associated with Catalan-Daehee numbers and their applications. Revista de la Real Academia de Ciencias Exactas, Físicas y Naturales. Serie A. Matemáticas 2017, 111, 1071-1081. [CrossRef]

8. Kim, D.S.; Kim, T. Triple symmetric identities for $w$-Catalan polynomials. J. Korean Math. Soc. 2017, 54, $1243-1264$.

9. Kim, T.; Kwon, H.-I. Revisit symmetric identities for the $\lambda$-Catalan polynomials under the symmetry group of degree n. Proc. Jangjeon Math. Soc. 2016, 19, 711-716.

10. Kim, T. A note on Catalan numbers associated with $p$-adic integral on Zp. Proc. Jangjeon Math. Soc. 2016, 19, 493-501.

11. Basic, B. On quotients of values of Euler's function on the Catalan numbers. J. Number Theory 2016, 169, 160-173. [CrossRef]

12. Qi, F.; Shi, X.T.; Liu, F.F. An integral representation, complete monotonicity, and inequalities of the Catalan numbers. Filomat 2018, 32, 575-587. [CrossRef]

13. Qi, F.; Shi, X.T.; Mahmoud, M. The Catalan numbers: A generalization, an exponential representation, and some properties. J. Comput. Anal. Appl. 2017, 23, 937-944.

14. Qi, F.; Guo, B.N. Integral representations of the Catalan numbers and their applications. Mathematics 2017, 5, 40. [CrossRef]

15. Aker, K.; Gursoy, A.E. A new combinatorial identity for Catalan numbers. Ars Comb. 2017, 135, 391-398.

16. Dolgy, D.V.; Jang, G.-W.; Kim, D.S.; Kim, T. Explicit expressions for Catalan-Daehee numbers. Proc. Jangjeon Math. Soc. 2017, 20, 1-9.

17. Ma, Y.K.; Zhang, W.P. Some identities involving Fibonacci polynomials and Fibonacci numbers. Mathematics 2018, 6, 334. [CrossRef]

18. Zhang, W.P.; Lin, X. A new sequence and its some congruence properties. Symmetry 2018, 10, 359. [CrossRef]

19. Zhang, Y.X.; Chen, Z.Y. A new identity involving the Chebyshev polynomials. Mathematics 2018, 6, 244. [CrossRef]

20. Zhao, J.H.; Chen, Z.Y. Some symmetric identities involving Fubini polynomials and Euler numbers. Symmetry 2018, 10, 303.

21. Zhang, W.P. Some identities involving the Fibonacci numbers and Lucas numbers. Fibonacci $Q$. 2004, 42, 149-154.

22. Guariglia, E. Entropy and Fractal Antennas. Entropy 2016, 18, 84. [CrossRef]

23. Guido, R.C. Practical and useful tips on discrete wavelet transforms. IEEE Signal Process. Mag. 2015, 32, 162-166. [CrossRef]

24. Berry, M.V.; Lewis, Z.V. On the Weierstrass-Mandelbrot fractal function. Proc. R. Soc. Lond. Ser. A 1980, 370, 459-484. [CrossRef]

25. Guido, R.C.; Addison, P.; Walker, J. Introducing wavelets and time-frequency analysis. IEEE Eng. Biol. Med. Mag. 2009, 28, 13. [CrossRef] [PubMed]

26. Guariglia, E. Harmonic Sierpinski Gasket and Applications. Entropy 2018, 20, 714. [CrossRef]

27. Zhang, W.P.; Chen, L. On the Catalan numbers and some of their identities. Symmetry 2019, 11, 62. [CrossRef]

(c) 2019 by the authors. Licensee MDPI, Basel, Switzerland. This article is an open access article distributed under the terms and conditions of the Creative Commons Attribution (CC BY) license (http:/ / creativecommons.org/licenses/by/4.0/). 